\title{
External symmetry in general relativity
}

\author{
Ion I. Cotăescu \\ The West University of Timişoara, \\ V. Parvan Ave. 4, RO-1900 Timişoara
}

August 29, 2021

\begin{abstract}
We propose a generalization of the isometry transformations to the geometric context of the field theories with spin where the local frames are explicitly involved. We define the external symmetry transformations as isometries combined with suitable tetrad gauge transformations and we show that these form a group which is locally isomorphic with the isometry one. We point out that the symmetry transformations that leave invariant the equations of the fields with spin have generators with specific spin terms which represent new physical observables. The examples we present are the generators of the central symmetry and those of the maximal symmetries of the de Sitter and anti-de Sitter spacetimes derived in different tetrad gauge fixings.

Pacs: 04.20.Cv, 04.62..+v, 11.30.-j
\end{abstract}

\section{Introduction}

In general relativity [1, 2, 3] the development of the quantum field theory in curved spacetimes [四] give rise to many difficult problems related to the physical interpretation of the one-particle quantum modes that may indicate how to quantize the field. This is because the form and the properties of the particular solutions of the free field equations, in the cases when these 
can be analytically solved [5, 6], are strongly dependent on the procedure of separation of variables and, implicitly, on the choice of the local chart. Moreover, when the fields have spin the situation is more complicated since then the field equations and, therefore, the form of their particular solutions depend, in addition, on the tetrad gauge in which one works [7, [1]. In these conditions it would be helpful to use the traditional method of the quantum theory in flat spacetime based on the complete sets of commuting operators that determine the quantum modes as common eigenstates and give physical meaning to the constants of the separation of variables which are just the eigenvalues of these operators. A good step in this direction could be to proceed like in special relativity looking for the generators of the geometric symmetries similar to the familiar momentum, angular momentum and spin operators of the Poincaré covariant field theories [8].

However, the relativistic covariance in the sense of general relativity is too general to play the same role as the Lorentz or Poincaré covariance in special relativity. In other respects, the tetrad gauge invariance of the theories with spin represents another kind of general symmetry that is not able to produce itself conserved observables [1]. For this reason we have to concentrate only upon some special transformations which should form a well-defined Lie group with significant parameterization from the geometric point of view. Obviously, these must be just the isometry transformations that point out the symmetry of the background giving us the specific Killing vectors [1, 3, 91. The physical fields take over this symmetry transforming according to appropriate representations of the isometry group. In the case of the scalar vector or tensor fields these representations are completely defined by the well-known transformations rules under coordinate transformations since the isometries are in fact particular automorphysms. It remains open the problem of the behavior under isometries of the fields with half integer spin which explicitly depend on the tetrad gauge fixing. Another important problem is how to define the generators of these representations for any spin. It is known that there is a standard operator-valued representation of the isometry group in the space of scalar functions whose generators can be written with the help of the Killing vectors in a similar manner as the orbital angular momentum operators of special relativity. Then it is natural to ask how could be defined the corresponding spin parts of the generators of the representations according which the fields with spin may transform.

Our aim here is to propose a way to solve these problems. We start with 
the idea that if we intend to study the symmetry of a physical theory we must take into consideration the whole geometric context, including the positions of the local frames given by the tetrad fields, since the spin is measured just with respect to the axes of these frames. Therefore, the symmetry transformations must preserve not only the form of the metric tensor but the tetrad gauge too. These have to be isometries combined with suitable tetrad gauge transformations in such a manner to leave invariant the tetrad field components. Thus we define the external symmetry group and we show that this is locally isomorphic with the isometry group, having the same structure constants. Moreover, there are arguments that in fact this is isomorphic with the universal covering group of the isometry one.

The next step is to define the operator-valued representations of the external symmetry group carried by spaces of fields with spin. We point out that these are induced by the linear finite-dimensional representations of the $S L(2, C)$ group. This is the motive why the symmetry transformations which leave invariant the field equations have generators with a composite structure. These have the usual orbital terms of the scalar representation and, in addition, specific spin terms which depend on the choice of the tetrad gauge even in the case of the fields with integer spin. In general, the spin and orbital terms do not commute to each other apart of some special gauge fixings where the fields transform manifestly covariant under external symmetry transformations.

These general results allow us to study two important examples, namely the central symmetry and the maximal symmetry of the de Sitter (dS) and anti-de Sitter (AdS) spacetimes. In the case of the central geometries we use central charts with Cartesian coordinates and the Cartesian tetrad gauge which allowed us recently to find new analytical solutions of the Dirac equation [10]. We show that in this gauge fixing the central symmetry becomes global and, consequently, the spin terms of its generators are the same as those of special relativity [11, 12]. This is important from the technical point of view since in the largely used diagonal tetrad gauge in spherical coordinates [13, 14] we obtain that the spin terms are partially hidden. For the $\mathrm{dS}$ and $\mathrm{AdS}$ spacetimes we calculate the generators of the representations of the external symmetry group in central charts with our Cartesian gauge and in Minkowskian charts [四] with another gauge where the fields behave manifestly covariant under the Lorentz symmetry [15].

We start in the second section with a brief review of the relativistic co- 
variance and gauge symmetry which will be treated together introducing the group of the combined transformations defined as gauge transformations followed by authomorphysms. The next section is devoted to our approach. Therein we define the external symmetry transformations, we show that these form a group and we study the operator-valued representations of this group and its Lie algebra. In Sec. 4 and 5 we discuss the mentioned examples.

We present our proposal at the level of the relativistic quantum mechanics in the sense of general relativity avoiding to consider the specific problems of the quantum field theory or to use too complicated mathematical methods. We work in natural units with $\hbar=c=1$.

\section{Relativistic covariance}

In the Lagrangian field theory in curved spacetimes the relativistic covariant equations of scalar, vector or tensor fields arise from actions that are invariant under general coordinate transformations. Moreover, when the fields have spin in the sense of the $S L(2, C)$ symmetry then the action must be invariant under tetrad gauge transformations [7]. The first step to our approach we propose here is to embed both these kind of transformations into new ones, called combined transformations, that will help us to understand the relativistic covariance in its most general terms.

\subsection{Gauge transformations}

Let us consider the curved spacetime $M$ and a local chart (natural frame) of coordinates $x^{\mu}, \mu=0,1,2,3$. Given a gauge, we denote by $e_{\hat{\mu}}(x)$ the tetrad fields that define the local frames, in each point $x$, and by $\hat{e}^{\hat{\mu}}(x)$ those of the corresponding coframes. These have the usual orthonormalization properties

$$
\hat{e}_{\alpha}^{\hat{\mu}} e_{\hat{\nu}}^{\alpha}=\delta_{\hat{\nu}}^{\hat{\mu}}, \quad \hat{e}_{\alpha}^{\hat{\mu}} e_{\hat{\mu}}^{\beta}=\delta_{\alpha}^{\beta}, \quad e_{\hat{\mu}} \cdot e_{\hat{\nu}}=\eta_{\hat{\mu} \hat{\nu}}, \quad \hat{e}^{\hat{\mu}} \cdot \hat{e}^{\hat{\nu}}=\eta^{\hat{\mu} \hat{\nu}},
$$

where $\eta=\operatorname{diag}(1,-1,-1,-1)$ is the Minkowki metric. From the line element

$$
d s^{2}=\eta_{\hat{\mu} \hat{\nu}} d \hat{x}^{\hat{\mu}} d \hat{x}^{\hat{\nu}}=g_{\mu \nu}(x) d x^{\mu} d x^{\nu},
$$


expressed in terms of 1 -forms, $d \hat{x}^{\hat{\mu}}=\hat{e}_{\nu}^{\hat{\mu}} d x^{\nu}$, we get the components of the metric tensor of the natural frame,

$$
g_{\mu \nu}=\eta_{\hat{\alpha} \hat{\beta}} \hat{e}_{\mu}^{\hat{\alpha}} \hat{e}_{\nu}^{\hat{\beta}}, \quad g^{\mu \nu}=\eta^{\hat{\alpha} \hat{\beta}} e_{\hat{\alpha}}^{\mu} e_{\hat{\beta}}^{\nu} .
$$

These raise or lower the natural vector indices, i.e., the Greek ones ranging from 0 to 3, while for the local vector indices, denoted by hat Greeks and having the same range, we must use the Minkowski metric. The derivatives $\hat{\partial}_{\hat{\nu}}=e_{\hat{\nu}}^{\mu} \partial_{\mu}$ satisfy the commutation rules

$$
\left[\hat{\partial}_{\hat{\mu}}, \hat{\partial}_{\hat{\nu}}\right]=e_{\hat{\mu}}^{\alpha} e_{\hat{\nu}}^{\beta}\left(\hat{e}_{\alpha, \beta}^{\hat{\sigma}}-\hat{e}_{\beta, \alpha}^{\hat{\sigma}}\right) \hat{\partial}_{\hat{\sigma}}=C_{\hat{\mu} \hat{\nu} \cdot \hat{\sigma}}^{\cdot} \hat{\partial}_{\hat{\sigma}}
$$

defining the Cartan coefficients which halp us to write the conecttion components in the local frames as

$$
\hat{\Gamma}_{\hat{\mu} \hat{\nu}}^{\hat{\sigma}}=e_{\hat{\mu}}^{\alpha} e_{\hat{\nu}}^{\beta}\left(\hat{e}_{\gamma}^{\hat{\sigma}} \Gamma_{\alpha \beta}^{\gamma}-\hat{e}_{\beta, \alpha}^{\hat{\sigma}}\right)=\frac{1}{2} \eta^{\hat{\sigma} \hat{\lambda}}\left(C_{\hat{\mu} \hat{\nu} \hat{\lambda}}+C_{\hat{\lambda} \hat{\mu} \hat{\nu}}+C_{\hat{\lambda} \hat{\nu} \hat{\mu}}\right)
$$

The notation $\Gamma_{\alpha \beta}^{\gamma}$ stands for the usual Christoffel symbols involved in the formulas of the covariant derivatives $\nabla_{\mu}={ }_{; \mu}$.

The Minkowski metric $\eta_{\hat{\mu} \hat{\nu}}$ remains invariant under the transformations of the gauge group of this metric, $G(\eta)=O(3,1)$. This has as subgroup the Lorentz group, $L_{+}^{\uparrow}$, of the transformations $\Lambda[A(\omega)]$ corresponding to the transformations $A(\omega) \in S L(2, C)$ through the canonical homomorphism [ 8 ]. In the standard covariant parameterization, with the real parameters $\omega^{\hat{\alpha} \hat{\beta}}=$ $-\omega^{\hat{\beta} \hat{\alpha}}$, we have

$$
A(\omega)=e^{-\frac{i}{2} \omega^{\hat{\alpha} \hat{\beta}} S_{\hat{\alpha} \hat{\beta}}}
$$

where $S_{\hat{\alpha} \hat{\beta}}$ are the covariant basis-generators of the $\operatorname{sl}(2, C)$ Lie algebra which satisfy

$$
\left[S_{\hat{\mu} \hat{\nu}}, S_{\hat{\sigma} \hat{\tau}}\right]=i\left(\eta_{\hat{\mu} \hat{\tau}} S_{\hat{\nu} \hat{\sigma}}-\eta_{\hat{\mu} \hat{\sigma}} S_{\hat{\nu} \hat{\tau}}+\eta_{\hat{\nu} \hat{\sigma}} S_{\hat{\mu} \hat{\tau}}-\eta_{\hat{\nu} \hat{\tau}} S_{\hat{\mu} \hat{\sigma}}\right)
$$

For small values of $\omega^{\hat{\alpha} \hat{\beta}}$ the matrix elements of the transformations $\Lambda$ can be written as

$$
\Lambda[A(\omega)]_{\cdot \hat{\nu}}^{\hat{\mu} \cdot}=\delta_{\hat{\nu}}^{\hat{\mu}}+\omega_{\cdot \hat{\nu}}^{\hat{\mu} \cdot}+\cdots
$$

Now we assume that $M$ is orientable and time-orientable such that $L_{+}^{\uparrow}$ can be considered as the gauge group of the Minkowski metric [3]. In these conditions the fields with spin can be defined as in the case of the flat spacetime, with the help of the finite-dimensional linear representations of the 
$S L(2, C)$ group [8]. Then any field $\psi_{\rho}: M \rightarrow V_{\rho}$ is defined over $M$ with values in the vector space $V_{\rho}$ of the representation $\rho$ which determines the spin content of $\psi_{\rho}$. In the following we systematically use the bases of $V_{\rho}$ labeled only by spinor or vector local indices defined with respect to the axes of the local frames given by the tetrad fields. Generally, these will not be written explicitly apart the cases when this is requested by the concrete calculation needs.

The relativistic covariant field equations are derived from actions [7, 1],

$$
\mathcal{S}\left[\psi_{\rho}, e\right]=\int d^{4} x \sqrt{g} \mathcal{L}\left(\psi_{\rho}, D_{\hat{\mu}} \psi_{\rho}\right), \quad g=\left|\operatorname{det}\left(g_{\mu \nu}\right)\right|
$$

with covariant derivatives,

$$
D_{\hat{\alpha}}=\hat{\partial}_{\hat{\alpha}}+\frac{i}{2} \rho\left(S_{\cdot \hat{\gamma}}^{\hat{\beta} \cdot}\right) \hat{\Gamma}_{\hat{\alpha} \hat{\beta}}^{\hat{\gamma}}
$$

that assure the invariance of the whole theory under the gauge transformations,

$$
\begin{aligned}
& \hat{e}_{\mu}^{\hat{\alpha}}(x) \quad \rightarrow \quad \hat{e}_{\mu}^{\hat{\alpha}}(x)=\Lambda[A(x)]_{\cdot \hat{\beta}}^{\hat{\alpha} \cdot} \hat{e}_{\mu}^{\hat{\beta}}(x), \\
& e_{\hat{\alpha}}^{\mu}(x) \rightarrow e_{\hat{\alpha}}^{\prime \mu}(x)=\Lambda[A(x)]_{\hat{\alpha} \cdot \hat{\beta}}^{\cdot} \cdot e_{\hat{\beta}}^{\mu}(x), \\
& \psi_{\rho}(x) \rightarrow \psi_{\rho}^{\prime}(x)=\rho\left[A(x) \psi_{\rho}(x),\right.
\end{aligned}
$$

determined by the mappings $A: M \rightarrow S L(2, C)$ the values of which are the local $S L(2, C)$ transformations $A(x) \equiv A[\omega(x)]$. These mappings can be organized as a group, $\mathcal{G}$, with respect to the multiplication $\times$ defined as $\left(A^{\prime} \times A\right)(x)=A^{\prime}(x) A(x)$. The notation $I d$ stands for the mapping identity, $\operatorname{Id}(x)=1 \in S L(2, C)$, while $A^{-1}$ is the inverse of $A,\left(A^{-1}\right)(x)=[A(x)]^{-1}$.

\subsection{Combined transformations}

The general coordinate transformations are automorphysms of $M$ which, in the passive mode, can be seen as changes of the local charts corresponding to the same domain of $M$ [3, 9]. If $x$ and $x^{\prime}$ are the coordinates of a point in two different charts then there is a mapping $\phi$ between these charts giving the coordinate transformation, $x \rightarrow x^{\prime}=\phi(x)$. These transformations form 
a group with respect to the composition of mappings, o, defined as usual, i.e. $\left(\phi^{\prime} \circ \phi\right)(x)=\phi^{\prime}[\phi(x)]$. We denote this group by $\mathcal{A}$, its identity map by $i d$ and the inverse mapping of $\phi$ by $\phi^{-1}$.

The automothysms change all the components carrying natural indices including those of the tetrad fields [1]. This means that, from the physical point of view, these transformations may change the positions of the local frames with respect to the natural ones. If we assume that the physical experiment makes reference to the axes of the local frame then it could appear situations when several correction of the positions of the local frames should be needed before (or after) each general coordinate transformation. Obviously, these have to be done with the help of suitable gauge transformation associated to the authomorphysms. Thus we arrive to the necessity of introducing the combined transformations denoted by $(A, \phi)$ and defined as gauge transformations, given by $A \in \mathcal{G}$, followed by automorphysms, $\phi \in \mathcal{A}$. In this new notation the pure gauge transformations will appear as $(A, i d)$ while the automorphysms will be denoted from now by $(I d, \phi)$.

The effect of a combined transformation $(A, \phi)$ upon our basic fields, $\psi_{\rho}, e$ and $\hat{e}$ is $x \rightarrow x^{\prime}=\phi(x), e(x) \rightarrow e^{\prime}\left(x^{\prime}\right), \hat{e}(x) \rightarrow \hat{e}^{\prime}\left(x^{\prime}\right)$ and $\psi_{\rho}(x) \rightarrow \psi_{\rho}^{\prime}\left(x^{\prime}\right)=$ $\rho[A(x)] \psi_{\rho}(x)$ where $e^{\prime}$ are the transformed tetrads of the components

$$
e_{\hat{\alpha}}^{\prime \mu}[\phi(x)]=\Lambda[A(x)]_{\hat{\alpha} \cdot}^{\hat{\beta}} e_{\hat{\beta}}^{\nu}(x) \frac{\partial \phi^{\mu}(x)}{\partial x^{\nu}},
$$

while the components of $\hat{e}^{\prime}$ have to be calculated according to Eqs.(11). Thus we have written down the most general transformation laws that leave invariant the action in the sense that $\mathcal{S}\left[\psi_{\rho}^{\prime}, e^{\prime}\right]=\mathcal{S}\left[\psi_{\rho}, e\right]$. The field equations derived from $\mathcal{S}$, written in operator form as $\left(E_{\rho} \psi_{\rho}\right)(x)=0$, covariantly transform under these transformations,

$$
\left(E_{\rho} \psi_{\rho}\right)(x) \rightarrow\left(E_{\rho}^{\prime} \psi_{\rho}^{\prime}\right)\left(x^{\prime}\right)=\rho[A(x)]\left(E_{\rho} \psi_{\rho}\right)(x)
$$

since the operators $E_{\rho}$ involve covariant derivatives [1].

The association among the transformations of the groups $\mathcal{G}$ and $\mathcal{A}$ must lead to a new group with a specific multiplication. In order to find how looks this new operation it is convenient to introduce the compositions among the mappings $A$ and $\phi$ (taken only in this order) giving new mappings, $A \circ \phi$, defined as $(A \circ \phi)(x)=A[\phi(x)]$. The calculation rules $I d \circ \phi=I d, A \circ i d=A$ and $\left(A^{\prime} \times A\right) \circ \phi=\left(A^{\prime} \circ \phi\right) \times(A \circ \phi)$ are obvious. With these ingredients we 
define the new multiplication

$$
\left(A^{\prime}, \phi^{\prime}\right) *(A, \phi)=\left(\left(A^{\prime} \circ \phi\right) \times A, \phi^{\prime} \circ \phi\right) .
$$

It is clear that now the identity is $(I d, i d)$ while the inverse of a pair $(A, \phi)$ reads

$$
(A, \phi)^{-1}=\left(A^{-1} \circ \phi^{-1}, \phi^{-1}\right) .
$$

First of all we observe that the operation $*$ is well-defined and represents the composition among the combined transformations since these can be expressed, according to their definition, as $(A, \phi)=(I d, \phi) *(A, i d)$. Furthermore, we can convince ourselves that if we perform successively two arbitrary combined transformations, $(A, \phi)$ and $\left(A^{\prime}, \phi^{\prime}\right)$, then the resulting transformation is just $\left(A^{\prime}, \phi^{\prime}\right) *(A, \phi)$ as given by Eq.(14). This means that the combined transformations form a group with respect to the multiplication $*$. It is not difficult to verify that this group, denoted by $\tilde{\mathcal{G}}$, is the semidirect product $\tilde{\mathcal{G}}=\mathcal{G}(\mathrm{S} \mathcal{A}$ where $\mathcal{G}$ is the invariant subgroup while $\mathcal{A}$ is an usual one.

In the theories involving only vector and tensor fields we do not need to use the combined transformations defined above since the theory is independent on the positions of the local frames. This can be easily shown even in our approach where we use field components with local indices. Indeed, if we perform a combined transformation $(A, \phi)$ then any tensor field of rank $(p, q)$

$$
\psi_{\hat{\beta}_{1}, \hat{\beta}_{2}, \ldots, \hat{\beta}_{q}}^{\hat{\alpha}_{1}, \hat{\alpha}_{2}, \ldots, \hat{\alpha}_{p}}=\hat{e}_{\mu_{1}}^{\hat{\alpha}_{1}} \cdots \hat{e}_{\mu_{p}}^{\hat{\alpha}_{p}} e_{\hat{\beta}_{1}}^{\nu_{1}} \cdots e_{\hat{\beta}_{q}}^{\nu_{q}} \psi_{\nu_{1}, \nu_{2}, \ldots, \nu_{q}}^{\mu_{1}, \mu_{2}, \ldots, \mu_{p}},
$$

transforms according to the representation

$$
\rho_{\hat{\alpha}_{1}, \hat{\alpha}_{2}, \ldots, \hat{\alpha}_{p} ; \hat{\beta}_{1}^{\prime}, \hat{\beta}_{2}^{\prime}, \ldots, \hat{\beta}_{q}^{\prime}}^{\hat{\beta}_{1}, \hat{\beta}_{2}, \hat{\beta}_{q}, \hat{\alpha}^{\prime}, \hat{\alpha}^{\prime}, \hat{\alpha}_{p}^{\prime}}(A)=\Lambda_{\cdot \hat{\beta}_{1}^{\prime}}^{\hat{\beta}_{1}} \cdot(A) \cdots \Lambda_{\hat{\alpha}_{1} \cdot}^{\cdot \hat{\alpha}_{1}^{\prime}}(A) \cdots,
$$

such that the resulting transformation law of the components carrying natural indices,

$$
\psi_{\nu_{1}, \ldots}^{\prime \mu_{1}, \ldots}\left(x^{\prime}\right)=\frac{\partial x^{\prime \mu_{1}}}{\partial x^{\sigma_{1}}} \cdots \frac{\partial x^{\tau_{1}}}{\partial x^{\prime \nu_{1}}} \cdots \psi_{\tau_{1}, \ldots}^{\sigma_{1}, \ldots .}(x),
$$

is just the familiar one [1]. In other words, in this case the effect of the combined transformations reduces to that of their authomorohysms. However, when the half integer spin fields are involved this is no more true and we must use the combined transformations of $\tilde{\mathcal{G}}$ if we want to keep under control the positions of the local frames. 


\section{External symmetry}

In general, the symmetry of any manifold $M$ is given by its isometry group whose transformations leave invariant the metric tensor in any chart. The scalar field transforms under isometries according to the standard scalar representation generated by the orbital generators related to the Killing vectors of $M$ [1, 3, 9]. In the following we propose a possible generalization of this theory of symmetry to the fields with spin, defining the external symmetry group and its representations.

\subsection{Isometries}

There are conjectures when several coordinate transformations, $x \rightarrow x^{\prime}=$ $\phi_{\xi}(x)$, depend on $N$ independent real parameters, $\xi^{a}(a, b, c \ldots=1,2, \ldots, N)$, such that $\xi=0$ corresponds to the identity map, $\phi_{\xi=0}=i d$. The set of these mappings can be organized as a Lie group [16], $G \in \mathcal{G}$, if they accomplish the composition rule

$$
\phi_{\xi^{\prime}} \circ \phi_{\xi}=\phi_{f\left(\xi^{\prime}, \xi\right)},
$$

where the functions $f: G \times G \rightarrow G$ define the group multiplication. These must satisfy $f^{a}(0, \xi)=f^{a}(\xi, 0)=\xi^{a}$ and $f^{a}\left(\xi^{-1}, \xi\right)=f^{a}\left(\xi, \xi^{-1}\right)=0$ where $\xi^{-1}$ are the parameters of the inverse mapping of $\phi_{\xi}, \phi_{\xi^{-1}}=\phi_{\xi}^{-1}$. Moreover, the structure constants of $G$ can be calculated as [17]

$$
c_{a b c}=\left(\frac{\partial f^{c}\left(\xi, \xi^{\prime}\right)}{\partial \xi^{a} \partial \xi^{\prime b}}-\frac{\partial f^{c}\left(\xi, \xi^{\prime}\right)}{\partial \xi^{b} \partial \xi^{\prime a}}\right)_{\mid \xi=\xi^{\prime}=0} .
$$

For small values of the group parameters the infinitesimal transformations, $x^{\mu} \rightarrow x^{\prime \mu}=x^{\mu}+\xi^{a} k_{a}^{\mu}(x)+\cdots$, are given by the vectors $k_{a}$ whose components,

$$
k_{a}^{\mu}={\frac{\partial \phi_{\xi}^{\mu}}{\partial \xi^{a}}}_{\mid \xi=0}
$$

satisfy the identities

$$
k_{a}^{\mu} k_{b, \mu}^{\nu}-k_{b}^{\mu} k_{a, \mu}^{\nu}+c_{a b c} k_{c}^{\nu}=0
$$


resulting from Eqs.(19) and (20).

In the following we restrict ourselves to consider only the isometry transformations, $x^{\prime}=\phi_{\xi}(x)$, which leave invariant the components of the metric tensor [1], 9], i.e.

$$
g_{\alpha \beta}\left(x^{\prime}\right) \frac{\partial x^{\prime \alpha}}{\partial x^{\mu}} \frac{\partial x^{\prime \beta}}{\partial x^{\nu}}=g_{\mu \nu}(x) .
$$

These form the isometry group $G \equiv I(M)$ which is the Lie group giving the symmetry of the spacetime $M$. We consider that this has $N$ independent parameters and, therefore, $k_{a}, a=1,2, \ldots N$, are independent Killing vectors (which satisfy $k_{a \mu ; \nu}+k_{a \nu ; \mu}=0$ ). Then their corresponding Lie derivatives form a basis of the Lie algebra $i(M)$ of the group $I(M)$ [9].

However, in practice we are interested to find the operators of the relativistic quantum theory related to these geometric objects which describe the symmetry of the background. For this reason we focus upon the operatorvalued representations [18] of the group $I(M)$ and its algebra. The scalar field $\psi: M \rightarrow C$ transforms under isometries as $\psi(x) \rightarrow \psi^{\prime}\left[\phi_{\xi}(x)\right]=\psi(x)$. This rule defines the representation $\phi_{\xi} \rightarrow T_{\xi}$ of the group $I(M)$ whose operators have the action $\psi^{\prime}=T_{\xi} \psi=\psi \circ \phi_{\xi}^{-1}$. Hereby it results that the operators of infinitesimal transformations, $T_{\xi}=1-i \xi^{a} L_{a}+\cdots$, depend on the basis-generators,

$$
L_{a}=-i k_{a}^{\mu} \partial_{\mu}, \quad a=1,2, \ldots, N,
$$

which are completely determined by the Killing vectors. From Eq.(22) we see that they obey the commutation rules

$$
\left[L_{a}, L_{b}\right]=i c_{a b c} L_{c},
$$

given by the structure constants of $I(M)$. In other words they form a basis of the operator-valued representation of the Lie algebra $i(M)$ in a carrier space of scalar fields. Notice that in the usual quantum mechanics the operators similar to the generators $L_{a}$ are called often orbital generators.

\subsection{The group of external symmetry}

Now the problem is how may transform under isometries the whole geo-

metric framework of the theories with spin where we explicitly use the local 
frames. Since the isometry is a general coordinate transformation it changes the relative positions of the local and natural frames. This fact may be an impediment when one intends to study the symmetries of the theories with spin induced by those of the background. For this reason it is natural to suppose that the good symmetry transformations we need are combined transformations in which the isometries are preceded by appropriate gauge transformations such that not only the form of the metric tensor should be conserved but the form of the tetrad field components too.

Thus we arrive at the main point of our proposal. We introduce the external symmetry transformations, $\left(A_{\xi}, \phi_{\xi}\right)$, as combined transformations involving isometries and corresponding gauge transformations necessary to preserve the gauge. We assume that in a fixed gauge, given by the tetrad fields $e$ and $\hat{e}, A_{\xi}$ is defined by

$$
\Lambda\left[A_{\xi}(x)\right]_{\cdot \hat{\beta}}^{\hat{\alpha} \cdot}=\hat{e}_{\mu}^{\hat{\alpha}}\left[\phi_{\xi}(x)\right] \frac{\partial \phi_{\xi}^{\mu}(x)}{\partial x^{\nu}} e_{\hat{\beta}}^{\nu}(x),
$$

with the supplementary condition $A_{\xi=0}(x)=1 \in S L(2, C)$. Since $\phi_{\xi}$ is an isometry Eq.(23) guarantees that $\Lambda\left[A_{\xi}(x)\right] \in L_{+}^{\uparrow}$ and, implicitly, $A_{\xi}(x) \in$ $S L(2, C)$. Then the transformation laws of our fields are

$$
\begin{aligned}
x & \rightarrow \quad x^{\prime}=\phi_{\xi}(x), \\
e(x) & \rightarrow e^{\prime}\left(x^{\prime}\right)=e\left[\phi_{\xi}(x)\right] \\
\hat{e}(x) & \rightarrow \hat{e}^{\prime}\left(x^{\prime}\right)=\hat{e}\left[\phi_{\xi}(x)\right] \\
\psi_{\rho}(x) & \rightarrow \psi_{\rho}^{\prime}\left(x^{\prime}\right)=\rho\left[A_{\xi}(x)\right] \psi_{\rho}(x) .
\end{aligned}
$$

The mean virtue of these transformations are that they leave invariant the operators of the field equations, $E_{\rho}$, since the components of the tetrad fields and, consequently, the covariant derivatives do not change their form.

For small $\xi^{a}$ the covariant $S L(2, C)$ parameters of $A_{\xi}(x) \equiv A\left[\omega_{\xi}(x)\right]$ can be written as $\omega_{\xi}^{\hat{\alpha} \hat{\beta}}(x)=\xi^{a} \Omega_{a}^{\hat{\alpha} \hat{\beta}}(x)+\cdots$ where, according to Eqs.(6), (贯) and (26), we have

$$
\Omega_{a}^{\hat{\alpha} \hat{\beta}} \equiv{\frac{\partial \omega_{\xi}^{\hat{\alpha} \hat{\beta}}}{\partial \xi^{a}}}_{\mid \xi=0}=\left(\hat{e}_{\mu}^{\hat{\alpha}} k_{a, \nu}^{\mu}+\hat{e}_{\nu, \mu}^{\hat{\alpha}} k_{a}^{\mu}\right) e_{\hat{\lambda}}^{\nu} \eta^{\hat{\lambda} \hat{\beta}}
$$

We must specify that these functions are antisymmetric if and only if $k_{a}$ are Killing vectors. This indicates that the association among isometries and the gauge transformations defined by Eq.(26) is correct. 
It remains to show that the transformations $\left(A_{\xi}, \phi_{\xi}\right)$ form a Lie group related to $I(M)$. Starting with Eq.(26) we find that

$$
\left(A_{\xi^{\prime}} \circ \phi_{\xi}\right) \times A_{\xi}=A_{f\left(\xi^{\prime}, \xi\right)}
$$

and, according to Eqs.(19) and (29), we obtain

$$
\left(A_{\xi^{\prime}}, \phi_{\xi^{\prime}}\right) *\left(A_{\xi}, \phi_{\xi}\right)=\left(A_{f\left(\xi^{\prime}, \xi\right)}, \phi_{f\left(\xi^{\prime}, \xi\right)}\right)
$$

and $\left(A_{\xi=0}, \phi_{\xi=0}\right)=(I d, i d)$. Thus we have shown that the pairs $\left(A_{\xi}, \phi_{\xi}\right)$ form a Lie group with respect to the operation $*$. We say that this is the external symmetry group of $M$ and we denote it by $S(M) \subset \tilde{\mathcal{G}}$. From Eq.(30) we understand that $S(M)$ is locally isomorphic with $I(M)$ and, therefore, the Lie algebra of $S(M)$, denoted by $s(M)$, is isomorphic with $i(M)$ having the same structure constants. In our opinion, $S(M)$ must be isomorphic with the universal covering group of $I(M)$ since it has anyway the topology induced by $S L(2, C)$ which is simply connected. In general, the number of group parameters of $I(M)$ or $S(M)$ (which is equal to the number of the independent Killing vectors of $M$ ) can be $0 \leq N \leq 10$.

The form of the external symmetry transformations is strongly dependent on the choice of the local chart as well as that of the tetrad gauge. If we change simultaneously the gauge and the coordinates with the help of a combined transformation $(A, \phi)$ then each $\left(A_{\xi}, \phi_{\xi}\right) \in S(M)$ transforms as

$$
\left(A_{\xi}, \phi_{\xi}\right) \rightarrow\left(A_{\xi}^{\prime}, \phi_{\xi}^{\prime}\right)=(A, \phi) *\left(A_{\xi}, \phi_{\xi}\right) *(A, \phi)^{-1}
$$

which means that

$$
\begin{aligned}
A_{\xi}^{\prime} & =\left\{\left[\left(A \circ \phi_{\xi}\right) \times A_{\xi}\right] \times A^{-1}\right\} \circ \phi^{-1} \\
\phi_{\xi}^{\prime} & =\left(\phi \circ \phi_{\xi}\right) \circ \phi^{-1} .
\end{aligned}
$$

\subsection{Representations}

The last of Eqs. 27) which gives the transformation law of the field $\psi_{\rho}$ defines the operator-valued representation $\left(A_{\xi}, \phi_{\xi}\right) \rightarrow T_{\xi}^{\rho}$ of the group $S(M)$,

$$
\left(T_{\xi}^{\rho} \psi_{\rho}\right)\left[\phi_{\xi}(x)\right]=\rho\left[A_{\xi}(x)\right] \psi_{\rho}(x) .
$$


The invariance of the field equations under these transformations requires to have

$$
T_{\xi}^{\rho} E_{\rho}\left(T_{\xi}^{\rho}\right)^{-1}=E_{\rho}
$$

Since $A_{\xi}(x) \in S L(2, C)$ we say that this representation is induced by the representation $\rho$ of $S L(2, C)$ 18, 19. As we have shown in Sec.2.2, if $\rho$ is a vector or tensor representation (having only integer spin components) then the effect of the transformation (34) upon the components carrying natural indices is due only to $\phi_{\xi}$. However, for the representations with half integer spin the presence of $A_{\xi}$ is crucial since there are no natural indices. Moreover, this allows us to define the generators of the representations (34) for any spin.

The basis-generators of the representations of the Lie algebra $s(M)$ are the operators

$$
X_{a}^{\rho}=i{\frac{\partial T_{\xi}^{\rho}}{\partial \xi^{a}}}_{\mid \xi=0}=S_{a}^{\rho}+L_{a},
$$

which appear as sums among the orbital generators defined by Eq.(24) and the spin terms which have the action

$$
\left(S_{a}^{\rho} \psi_{\rho}\right)(x)=\rho\left[S_{a}(x)\right] \psi_{\rho}(x)
$$

This is determined by the form of the $\operatorname{local} \operatorname{sl}(2, C)$ generators,

$$
S_{a}(x)=i{\frac{\partial A_{\xi}(x)}{\partial \xi^{a}}}_{\mid \xi=0}=\frac{1}{2} \Omega_{a}^{\hat{\alpha} \hat{\beta}}(x) S_{\hat{\alpha} \hat{\beta}},
$$

which depend on the functions (28). Furthermore, if we derive Eq.(29) with respect to $\xi$ and $\xi^{\prime}$ then from Eqs.(8), (20) and (28), after a few manipulations, we find the identities

$$
\eta_{\hat{\alpha} \hat{\beta}}\left(\Omega_{a}^{\hat{\alpha} \hat{\mu}} \Omega_{b}^{\hat{\beta} \hat{\nu}}-\Omega_{b}^{\hat{\alpha} \hat{\mu}} \Omega_{a}^{\hat{\beta} \hat{\nu}}\right)+k_{a}^{\mu} \Omega_{b, \mu}^{\hat{\mu} \hat{\nu}}-k_{b}^{\mu} \Omega_{a, \mu}^{\hat{\mu} \hat{\nu}}+c_{a b c} \Omega_{c}^{\hat{\mu} \hat{\nu}}=0 .
$$

Hereby it results that

$$
\left[S_{a}^{\rho}, S_{b}^{\rho}\right]+\left[L_{a}, S_{b}^{\rho}\right]-\left[L_{b}, S_{a}^{\rho}\right]=i c_{a b c} S_{c}^{\rho}
$$

and, according to Eq.(25), we find the expected commutation rules

$$
\left[X_{a}^{\rho}, X_{b}^{\rho}\right]=i c_{a b c} X_{c}^{\rho}
$$


Thus we have obtained the basis-generators of a operator-valued representation of $s(M)$ induced by the linear representation $\rho$ of $\operatorname{sl}(2, C)$. All the operators of this representation commute with the operator $E_{\rho}$ since, according to Eqs.(35) and (36), we have

$$
\left[E_{\rho}, X_{a}^{\rho}\right]=0, \quad a=1,2, \ldots, N .
$$

Therefore, for defining quantum modes we can use the set of commuting operators containing the Casimir operators those of the Cartan subalgebra and $E_{\rho}$.

The action of the operators $X_{a}^{\rho}$ depends on the choice of many elements: the natural coordinates, the tetrad gauge, the group parameterization and the representation $\rho$. What is important here is that they are strongly dependent on the tetrad gauge fixing even in the case of the representations with integer spin. This is because the covariant parameterization of the $s l(2, C)$ algebra is defined with respect to the axes of the local frames. In general, if we consider the representation $\left(A_{\xi}, \phi_{\xi}\right) \rightarrow T_{\xi}^{\rho}$ and we perform a combined transformation (31) then it results the equivalent representation, $\left(A_{\xi}^{\prime}, \phi_{\xi}^{\prime}\right) \rightarrow T_{\xi}^{\prime \rho}$. Its generators calculated from Eqs.(32) indicate that in this case the equivalence relations are much more complicated than those of the usual theory of linear representations. Without to enter in other technical details we specify that if we change only the gauge with the help of the transformation $(A, i d)$ then the local $s l(2, C)$ generators (38) transform as

$$
\begin{aligned}
S_{a}(x) \rightarrow S_{a}^{\prime}(x)= & A(x) S_{a}(x) A(x)^{-1} \\
& +k_{a}^{\sigma}(x) \Lambda[A(x)]_{\hat{\alpha} \hat{\mu}, \sigma} \Lambda[A(x)]_{\hat{\beta}}^{\cdot \hat{\mu}} S^{\hat{\alpha} \hat{\beta}},
\end{aligned}
$$

while the orbital parts do not change their form. This means that the gauge transformations change, in addition, the commutation relations among the spin and orbital parts of the generators $X_{a}^{\rho}$. Hence we can draw the conclusion that the choice of the tetrad gauge which defines the local frames may have important consequences upon the measurement of the local spin effects.

There are gauge fixings where the local $\operatorname{sl}(2, C)$ generators $S_{a}(x), a=$ $1,2, \ldots, n(n \leq N)$, corresponding to a subgroup $H \subset S(M)$, are independent on $x$ and, therefore, $\left[S_{a}^{\rho}, L_{b}\right]=0$ for all $a=1,2, \ldots, n$ and $b=1,2, \ldots, N$. Then the operators $S_{a}^{\rho}$ are just the basis-generators of an usual linear representation of $H$ and the field $\psi_{\rho}$ behaves manifestly covariant under the external 
symmetry transformations of this subgroup. Of course, when $H=S(M)$ we say simply that the field $\psi_{\rho}$ is manifest covariant.

The simplest examples are the manifest covariant fields of special relativity. Here since the spacetime $M$ is flat the metric in Cartesian coordinates is $g_{\mu \nu}=\eta_{\mu \nu}$ and one can use the inertial (local) frames with $e_{\nu}^{\mu}=\hat{e}_{\nu}^{\mu}=\delta_{\nu}^{\mu}$. Then the isometries are just the transformations $x^{\prime}=\Lambda[A(\omega)] x-a$ of the Poincaré group, $\mathcal{P}_{+}^{\uparrow}=T(4) \subseteq L_{+}^{\uparrow}$ [8]. If we denote by $\xi^{(\mu \nu)}=\omega^{\mu \nu}$ the $S L(2, C)$ parameters and by $\xi^{(\mu)}=a^{\mu}$ those of the translation group $T(4)$, then we find that $S(M) \equiv \tilde{\mathcal{P}}_{+}^{\uparrow}=T(4) \subseteq S L(2, C)$ is just the universal covering group of $\mathcal{P}_{+}^{\uparrow}$. Furthermore, it is a simple exercise to calculate the basis-generators

$$
\begin{aligned}
X_{(\mu)}^{\rho} & =i \partial_{\mu}, \\
X_{(\mu \nu)}^{\rho} & =\rho\left(S_{\mu \nu}\right)+i\left(\eta_{\mu \alpha} x^{\alpha} \partial_{\nu}-\eta_{\nu \alpha} x^{\alpha} \partial_{\mu}\right),
\end{aligned}
$$

which show us that $\psi_{\rho}$ transforms manifestly covariant.

In general, there are many cases of curved spacetimes for which one can choose suitable local frames allowing one to introduce manifest covariant fields with respect to a subgroup $H \subset S(M)$ or even the whole group $S(M)$. In our opinion, this is possible only when $H$ or $S(M)$ are at most subgroups of $\tilde{\mathcal{P}}_{+}^{\uparrow}$.

\section{The central symmetry}

Let us take as first example the spacetimes $M$ which have spherically symmetric static chart that will be referred here as central charts. These manifolds have the isometry group $I(M)=T(1) \otimes S O(3)$ of time translations and space rotations.

\subsection{Central charts}

In a central chart with Cartesian coordinates $x^{0}=t$ and $x^{i}(i, j, k \ldots=$ $1,2,3)$, the metric tensor is time-independent and transforms manifestly covariant under the rotations $R \in S O(3)$ of the space coordinates,

$$
t^{\prime}=t, \quad x^{i}=R_{\cdot j}^{i \cdot}(\omega) x^{j}=x^{i}+\omega_{\cdot j}^{i \cdot} x^{j}+\cdots,
$$


denoted simply by $x \rightarrow x^{\prime}=R x$. Here the most general form of the line element,

$$
d s^{2}=g_{\mu \nu}(x) d x^{\mu} d x^{\nu}=A(r) d t^{2}-\left[B(r) \delta_{i j}+C(r) x^{i} x^{j}\right] d x^{i} d x^{j},
$$

may involve three functions, $A, B$ and $C$, depending only on the Euclidean norm of $\vec{x}, r=|\vec{x}|$. In applications it is convenient to replace these functions by new ones, $u, v$ and $w$, defined as

$$
A=w^{2}, \quad B=\frac{w^{2}}{v^{2}}, \quad C=\frac{w^{2}}{r^{2}}\left(\frac{1}{u^{2}}-\frac{1}{v^{2}}\right) .
$$

Other useful central charts are those with spherical coordinates, $r, \theta, \phi$, commonly associated with the Cartesian space ones. Here the line elements are

$$
d s^{2}=w^{2} d t^{2}-\frac{w^{2}}{u^{2}} d r^{2}-\frac{w^{2}}{v^{2}} r^{2}\left(d \theta^{2}+\sin ^{2} \theta d \phi^{2}\right) .
$$

In these charts we see that the advantage of the new functions we have introduced is of simple transformation laws under the isotropic dilatations which change only the radial coordinate, $r \rightarrow r^{\prime}(r)$, without to affect the central symmetry of the line element. These transformations,

$$
u^{\prime}\left(r^{\prime}\right)=u(r)\left|\frac{d r^{\prime}(r)}{d r}\right|, \quad v^{\prime}\left(r^{\prime}\right)=v(r) \frac{r^{\prime}(r)}{r}, \quad w^{\prime}\left(r^{\prime}\right)=w(r),
$$

allow one to choose desired forms for the functions $u, v$ and $w$.

\subsection{The Cartesian gauge}

The Cartesian gauge in central charts was mentioned long time ago [13 but it is less used in concrete problems since it leads to complicated calculations in spherical coordinates. However, in Cartesian coordinates this gauge has the advantage of explicitly pointing out the global central symmetry of the manifold. In Refs. 10 we have proposed a version of Cartesian gauge in central charts with Cartesian coordinates that preserve the manifest covariance under rotations (46) in the sense that the corresponding 1-forms transform as

$$
d \hat{x}^{\hat{\mu}} \rightarrow d \hat{x}^{\prime \hat{\mu}}=\hat{e}_{\alpha}^{\hat{\mu}}\left(x^{\prime}\right) d x^{\prime \alpha}=(R d \hat{x})^{\hat{\mu}} .
$$


If the line element has the form (47) then the most general choice of the tetrad fields with the above property is

$$
\begin{array}{ll}
\hat{e}_{0}^{0}=\hat{a}(r), & \hat{e}_{i}^{0}=\hat{e}_{0}^{i}=0, \quad \hat{e}_{j}^{i}=\hat{b}(r) \delta_{i j}+\hat{c}(r) x^{i} x^{j}+\hat{d}(r) \epsilon_{i j k} x^{k} \\
e_{0}^{0}=a(r), & e_{i}^{0}=e_{0}^{i}=0, \quad e_{j}^{i}=b(r) \delta_{i j}+c(r) x^{i} x^{j}+d(r) \epsilon_{i j k} x^{k}
\end{array}
$$

where, according to (3), (47) and (48), we must have

$$
\begin{aligned}
& \hat{a}=w, \hat{b}=\frac{w}{v} \cos \alpha, \hat{c}=\frac{1}{r^{2}}\left(\frac{w}{u}-\frac{w}{v} \cos \alpha\right), \hat{d}=\frac{1}{r} \frac{w}{v} \sin \alpha, \\
& a=\frac{1}{w}, \quad b=\frac{v}{w} \cos \alpha, c=\frac{1}{r^{2}}\left(\frac{u}{w}-\frac{v}{w} \cos \alpha\right), d=-\frac{1}{r} \frac{v}{w} \sin \alpha .
\end{aligned}
$$

The angle $\alpha$ is an arbitrary function of $r$ which is not explicitly involved in the expression of the metric tensor since it represents the angle of an arbitrary rotation of the local frame around the direction of $\vec{x}$, that does not change the relative position of $\vec{x}$ with respect to this frame.

When one defines the metric tensor such that $g_{\mu \nu \mid r=0}=\eta_{\mu \nu}$ then $u(0)^{2}=$ $v(0)^{2}=w(0)^{2}=1$. Moreover, it is natural to take $\alpha(0)=0$. In other respects, from Eqs.(54) and (55) we see that the function $w$ must be positively defined in order to keep the same sense for the time axes of the natural and local frames. In addition, it is convenient to consider that the function $u$ is positively defined too. However, the function $v=\eta_{P}|v|$ has the sign given by the relative parity $\eta_{P}$ which takes the value $\eta_{P}=1$ when the space axes of the local frame at $x=0$ are parallel with those of the natural frame, and $\eta_{P}=-1$ if these are antiparalel.

Now we have all the elements we need to calculate the generators of the representations $T^{\rho}$ of the group $S(M)$. If we denote by $\xi^{(0)}$ the parameter of the time translations and by $\xi^{(i)}=\epsilon_{i j k} \omega^{j k} / 2$ the parameters of the rotations (46), we find that the local $s l(2, C)$ generators of Eq.(38) are just the $s u(2)$ ones, i.e. $S_{(i j)}(x)=S_{i j}$, such that the basis-generators read

$$
X_{(0)}^{\rho}=i \partial_{t}, \quad X_{(i)}^{\rho}=\frac{1}{2} \epsilon_{i j k} \rho\left(S_{j k}\right)+L_{(i)}
$$

where $L_{(i)}=-i \epsilon_{i j k} x^{j} \partial_{k}$ are the usual components of the orbital angular momentum. Thus we obtain that the group $S(M)=T(1) \otimes S U(2)$ is the universal covering group of $I(M)$. The physical significance of the basisgenerators is the usual one, namely $X_{(0)}^{\rho}$ is the Hamiltonian operator while 
$X_{(i)}^{\rho} \equiv J_{(i)}^{\rho}$ are the components of the whole angular momentum operator of the field $\psi_{\rho}$ which transforms manifestly covariant.

We can conclude that, in our Cartesian gauge, the local frames play the same role as the usual Cartesian rest frames of the central sources in flat spacetime since their axes are just those of projections of the angular momenta.

\subsection{The diagonal gauge}

In other gauge fixings the basis-generators are quite different. A tetrad gauge largely used in central charts with spherical coordinates [14] is the diagonal gauge defined by the 1 -forms

$$
d \hat{x}_{s}^{0}=w d t, \quad d \hat{x}_{s}^{1}=\frac{w}{u} d r, \quad d \hat{x}_{s}^{2}=r \frac{w}{v} d \theta, \quad d \hat{x}_{s}^{3}=r \frac{w}{v} \sin \theta d \phi .
$$

In this gauge the angular momentum operators of the canonical basis (where $\left.J_{( \pm)}=J_{(1)} \pm i J_{(2)}\right)$ are

$$
J_{( \pm)}^{\rho}=\frac{e^{ \pm i \phi}}{\sin \theta} \rho\left(S_{23}\right)+L_{( \pm)}, \quad J_{(3)}^{\rho}=L_{(3)} .
$$

Thus we obtain a representation of $S U(2)$ where the spin terms do not commute with the orbital ones and, therefore, the field $\psi_{\rho}$ does not transform manifestly covariant under rotations. In this case we can say that the spin part of the central symmetry remains partially hidden because of the diagonal gauge which determines special positions of the local frames with respect to the natural one. However, when this is an impediment one can change anytime this gauge into the Cartesian one by using a simple local rotation. For the flat spacetimes these transformations and their effects upon the Dirac equation are studied in Ref.[20]. We note that the form of the spin generators as well as that of the mentioned rotation depend on the enumeration of the 1-forms (57). 


\section{The dS and AdS symmetries}

The backgrounds with highest external symmetry are the dS and the AdS spacetimes. We shall briefly discuss simultaneously both these manifolds which will be denoted by $M_{\epsilon}$ where $\epsilon=1$ for dS case and $\epsilon=-1$ for AdS one. Our goal here is to calculate the generators of the representations of the group $S\left(M_{\epsilon}\right)$ induced by those of $S L(2, C)$.

The dS and AdS spacetimes are hyperboloids in the $(4+1)$ or $(3+2)$ dimensional flat spacetimes, $M_{\epsilon}^{5}$, of coordinates $Z^{A}, A, B, \ldots=0,1,2,3,5$, and the metric $\eta(\epsilon)=\operatorname{diag}(1,-1,-1,-1,-\epsilon)$. The equation of the hyperboloid of radius $r_{0}=1 / \hat{\omega}$ reads

$$
-\eta_{A B}(\epsilon) Z^{A} Z^{B}=\epsilon r_{0}^{2}
$$

From their definitions it results that the dS or AdS spacetimes are homogeneous spaces of the pseudo-orthogonal groups $S O(4,1)$ or $S O(3,2)$ which play the role of gauge groups of the metric $\eta(\epsilon)$ (for $\epsilon=1$ and $\epsilon=-1$ respectively) and represent just the isometry groups of these manifolds, $G[\eta(\epsilon)]=I\left(M_{\epsilon}\right)$. Then it is natural to use the covariant real parameters $\omega^{A B}=-\omega^{B A}$ since in this parameterization the orbital basis-generators of the representations of $G[\eta(\epsilon)]$ carried by the spaces of the functions over $M_{\epsilon}^{5}$ have the usual form

$$
L_{A B}^{5}=i\left[\eta_{A C}(\epsilon) Z^{C} \partial_{B}-\eta_{B C}(\epsilon) Z^{C} \partial_{A}\right] .
$$

They will give us directly the orbital basis-generators of the representations of $S\left(M_{\epsilon}\right)$ in the carrier spaces of the functions defined over dS or AdS spacetimes.

\subsection{Central charts}

The hyperboloid equation can be solved in Cartesian dS/AdS coordinates, $x^{0}=t$ and $x^{i}(i=1,2,3)$, which satisfy

$$
Z^{5}=\hat{\omega}^{-1} \chi_{\epsilon}(r) \begin{cases}\cosh \hat{\omega} t & \text { if } \epsilon=1 \\ \cos \hat{\omega} t & \text { if } \epsilon=-1\end{cases}
$$




$$
\begin{aligned}
& Z^{0}=\hat{\omega}^{-1} \chi_{\epsilon}(r) \begin{cases}\sinh \hat{\omega} t & \text { if } \epsilon=1 \\
\sin \hat{\omega} t & \text { if } \epsilon=-1\end{cases} \\
& Z^{i}=x^{i},
\end{aligned}
$$

where we have denoted $\chi_{\epsilon}(r)=\sqrt{1-\epsilon \omega^{2} r^{2}}$. The line elements

$$
\begin{aligned}
d s^{2} & =\eta_{A B}(\epsilon) d Z^{A} d Z^{B} \\
& =\chi_{\epsilon}(r)^{2} d t^{2}-\frac{d r^{2}}{\chi_{\epsilon}(r)^{2}}-r^{2}\left(d \theta^{2}+\sin ^{2} \theta d \phi^{2}\right)
\end{aligned}
$$

are defined on the radial domains $D_{r}=[0,1 / \sqrt{\hat{\omega}})$ or $D_{r}=[0, \infty)$ for $\mathrm{dS}$ or AdS respectively.

We calculate the Killing vectors and the orbital generators of the external symmetry in the Cartesian coordinates defined by Eq.(61) and the mentioned parameterization of $I\left(M_{\epsilon}\right)$ starting with the identification $\xi^{(A B)}=\omega^{A B}$. Then, from Eqs.(24) and (60), after a few manipulations, we obtain the orbital basis-generators

$$
\begin{aligned}
L_{(05)} & =\frac{i \epsilon}{\hat{\omega}} \partial_{t}, \\
L_{(j 5)} & =\frac{i \epsilon}{\hat{\omega}} \chi_{\epsilon}(r)\left(\begin{array}{c}
\cosh \hat{\omega} t \\
\cos \hat{\omega} t
\end{array}\right) \partial_{j}+\frac{i x^{j}}{\chi_{\epsilon}(r)}\left(\begin{array}{c}
\sinh \hat{\omega} t \\
\sin \hat{\omega} t
\end{array}\right) \partial_{t}, \\
L_{(0 j)} & =\frac{i}{\hat{\omega}} \chi_{\epsilon}(r)\left(\begin{array}{c}
\sinh \hat{\omega} t \\
\sin \hat{\omega} t
\end{array}\right) \partial_{j}+\frac{i x^{j}}{\chi_{\epsilon}(r)}\left(\begin{array}{c}
\cosh \hat{\omega} t \\
\cos \hat{\omega} t
\end{array}\right) \partial_{t}, \\
L_{(i j)} & =-i\left(x^{i} \partial_{j}-x^{j} \partial_{i}\right) .
\end{aligned}
$$

Furthermore, we consider the Cartesian tetrad gauge defined by Eqs.(52) (55) where, according to Eq.(62), we have

$$
u(r)=\chi_{\epsilon}(r)^{2}, \quad v(r)=w(r)=\chi_{\epsilon}(r) .
$$

In addition we take $\alpha=0$. In this gauge we obtain the following local $s l(2, C)$ generators

$$
\begin{aligned}
& S_{(05)}(x)=0, \\
& S_{(j 5)}(x)=S_{0 j}\left(\begin{array}{l}
\sinh \hat{\omega} t \\
\sin \hat{\omega} t
\end{array}\right)+\frac{1}{r^{2}}\left[\chi_{\epsilon}(r)-1\right]\left[\epsilon \frac{S_{j k} x^{k}}{\hat{\omega}}\left(\begin{array}{l}
\cosh \hat{\omega} t \\
\cos \hat{\omega} t
\end{array}\right)\right.
\end{aligned}
$$




$$
\begin{aligned}
& \left.-\frac{S_{0 k} x^{k} x^{j}}{\chi_{\epsilon}(r)}\left(\begin{array}{l}
\sinh \hat{\omega} t \\
\sin \hat{\omega} t
\end{array}\right)\right], \\
S_{(0 j)}(x)= & S_{0 j}\left(\begin{array}{c}
\cosh \hat{\omega} t \\
\cos \hat{\omega} t
\end{array}\right)+\frac{1}{r^{2}}\left[\chi_{\epsilon}(r)-1\right]\left[\frac{S_{j k} x^{k}}{\hat{\omega}}\left(\begin{array}{c}
\sinh \hat{\omega} t \\
\sin \hat{\omega} t
\end{array}\right)\right. \\
& \left.-\frac{S_{0 k} x^{k} x^{j}}{\chi_{\epsilon}(r)}\left(\begin{array}{l}
\cosh \hat{\omega} t \\
\cos \hat{\omega} t
\end{array}\right)\right], \\
S_{(i j)}(x)= & S_{i j} .
\end{aligned}
$$

With their help we can write the action of the spin terms (37) and, implicitly, that of the basis-generators $X_{(A B)}^{\rho}=S_{(A B)}^{\rho}+L_{(A B)}$ of the representations of $S\left(M_{\epsilon}\right)$ induced by the representations $\rho$ of $S L(2, C)$. Hereby it is not difficult to show that $S\left(M_{\epsilon}\right)$ is isomorphic with the universal covering group of $I\left(M_{\epsilon}\right)$ which in both cases $(\epsilon= \pm 1)$ is a subgroup of the $S U(2,2)$ group. As was expected, in the central charts and Cartesian gauge the fields transform manifestly covariant only under the transformations of the subgroup $S U(2) \subset$ $S\left(M_{\epsilon}\right)$.

\subsection{Minkowskian charts}

Another possibility is to solve the hyperboloid equation (59) in Minkowskian charts [1] where the coordinates, $x^{\mu}$, are defined by

$$
Z^{5}=\hat{\omega}^{-1} \tilde{\chi}_{\epsilon}(s), \quad Z^{\mu}=x^{\mu},
$$

with $\tilde{\chi}_{\epsilon}(s)=\sqrt{1+\epsilon \hat{\omega}^{2} s^{2}}$ and $s^{2}=\eta_{\mu \nu} x^{\mu} x^{\nu}$. In these coordinates it is convenient to identify the hat indices with the usual ones and to do not rise or lower these indices. Then we find that the metric tensor,

$$
g_{\mu \nu}(x)=\eta_{\mu \nu}-\frac{\epsilon \hat{\omega}^{2}}{\tilde{\chi}_{\epsilon}(s)^{2}} \eta_{\mu \alpha} x^{\alpha} \eta_{\nu \beta} x^{\beta},
$$

transforms manifestly covariant under the global $L_{+}^{\uparrow}$ transformations, $x^{\prime \mu} \rightarrow$ $x^{\mu}=\Lambda_{\cdot \nu}^{\mu} \cdot x^{\nu}$. Moreover, the whole theory remains manifest covariant if we use the tetrad fields in the Lorentz gauge defined as [15]

$$
e_{\nu}^{\mu}(x)=\delta_{\nu}^{\mu}+h_{\epsilon}(s) \eta_{\nu \alpha} x^{\alpha} x^{\mu}, \quad \hat{e}_{\nu}^{\mu}(x)=\delta_{\nu}^{\mu}+\hat{h}_{\epsilon}(s) \eta_{\nu \alpha} x^{\alpha} x^{\mu},
$$


where

$$
h_{\epsilon}(s)=\frac{1}{s^{2}}\left[\tilde{\chi}_{\epsilon}(s)-1\right], \quad \hat{h}_{\epsilon}(s)=\frac{1}{s^{2}}\left[\frac{1}{\tilde{\chi}_{\epsilon}(s)}-1\right] .
$$

First we calculate the $S O(4,1)$ or $S O(3,2)$ orbital generators,

$$
\begin{aligned}
L_{(\mu 5)} & =\frac{i \epsilon}{\hat{\omega}} \tilde{\chi}_{\epsilon}(s) \partial_{\mu}, \\
L_{(\mu \nu)} & =i\left(\eta_{\mu \alpha} x^{\alpha} \partial_{\nu}-\eta_{\nu \alpha} x^{\alpha} \partial_{\mu}\right),
\end{aligned}
$$

which are independent on the gauge fixing. We observe that in the Minkowskian charts $\partial_{t}$ is no more a Killing vector as in the case of the central ones. However, here we have another advantage namely that of the Lorentz gauge in which the local $\operatorname{sl}(2, C)$ generators of Eq.(37) have the form

$$
\begin{aligned}
S_{(\mu 5)}(x) & =-\frac{\epsilon}{\hat{\omega} s^{2}}\left[\tilde{\chi}_{\epsilon}(s)-1\right] S_{\mu \alpha} x^{\alpha}, \\
S_{(\mu \nu)}(x) & =S_{\mu \nu},
\end{aligned}
$$

showing that the field $\psi_{\rho}$ transforms manifestly covariant under the whole $S L(2, C)$ subgroup of $S\left(M_{\epsilon}\right)$. Since these representations are induced just by those of $S L(2, C)$ we can say that in this gauge the manifest covariance is maximal.

\section{Concluding remarks}

We have presented here the theory of external symmetry in general relativity. Starting with the group $I(M)$ which gives the symmetry of the background, we have defined the group $S(M)$ showing that its Lie algebra, $s(M)$, is isomorphic to $i(M)$, having the same structure constants. We have pointed out that the fields with spin transform according to the representations of the $S(M)$ group induced by linear representations of $S L(2, C)$. This allowed us to calculate the generators of these representations which have specific spin terms. In this way we have obtained the operators associated to the external symmetries which commute with the operator of the relativistic covariant field equation. We have thus the opportunity to choose suitable sets of commuting operators which should determine the quantum modes. 
In other respects, since the concrete form of these generators depends on the choice of both the natural and local frames, the commutation rules among their spin and orbital parts are determined by the tetrad gauge. Consequently, the results of the local measurement of the spin observables may depend on the positions of the local frame. This suggests that it should be interesting to investigate new inertial spin effects in other possible tetrad gauge fixings.

However, in our opinion, the most important domain of the further developmens is that of the external symmetry of the quantum field theory in curved spacetimes where the generators of the symmetry transformations must be the one-particle operators corresponding to the external symmetries through the Noether theorem.

\section{Acknowledgments}

I would like to thank Mircea Bundaru and Mihai Visinescu for useful comments and enlightening discussions about some sensitive problems appearing here.

\section{References}

[1] S. Weinberg, Gravitation and Cosmology: Principles and Applications of the General Theory of Relativity (Wiley, New York, 1972)

[2] C. M. Misner, K. S. Thorne and J. A. Wheeler, Gravitation (W. H. Freeman \& Co., San Francisco, 1973)

[3] R. M. Wald, General Relativity, (The Univ. of Chicago Press, Chicago and London, 1984)

[4] N. D. Birrel and P. C. W. Davies, Quantum Fields in Curved Space (Cambridge University Press, Cambridge 1982)

[5] S. J. Avis, C. J. Isham and D. Storey, Phys. Rev. D 10, 3565 (1978); P. Breitenlohner and D. Z. Freedman, Phys. Lett. 115B, 197 (1982); D. J. Navarro and J. Navarro-Salas, J. Math. Phys. 37, 6006 (1996); I. I. Cotăescu, Phys Rev. D 60, 107504 (1999) 
[6] V. S. Otchik, Class. Quant. Grav. 2, 539 (1985); L. P. Chimento and M. S. Mollerach, Phys. Rev. D 34, 3698 (1986); Phys. Lett. A 121, 7 (1987); M. A. Costagnino, C. D. El Hasi, F. D. Mozzitelli and J. P. Paz, Phys. Lett. A 128, 25 (1988) V. M. Villalba and U. Percoco, J. Math. Phys. 31, 715 (1990); G. V. Shishkin, Class. Quant. Grav. 8, 175 (1991); G. V. Shishkin and V. M. Villalba, J. Math. Phys. 30, 2132 (1989); J. Math. Phys. 33, 2093 (1992)

[7] R. Utiyama, Phys. Rev. 101, 1597 (1956); T. W. B. Kibble, J. Math. Phys. 2, 212 (1961)

[8] W.- K. Tung, Group Theory in Physics (World Sci., Philadelphia, 1985)

[9] B. O’Neill, Semi-Riemannian Geometry (Academic Press, 1983)

[10] I. I. Cotăescu, Mod. Phys. Lett. A 13, 2923 (1998); id 13, 2991 (1998); Phys. Rev. D 60, 124006 (1999)

[11] J. D. Bjorken and S. D. Drell S.D. Relativistic Quantum Mechanics (McGraw-Hill Book Co., NY, 1964)

[12] B. Thaller, The Dirac Equation (Springer Verlag, Berlin Heidelberg, 1992)

[13] D. R. Brill and J. A. Wheeler, Rev. Mod. Phys. 29, 465 (1957)

[14] D. R. Brill and J. A. Cohen, J. Math. Phys. 7, 238 (1966); J. Klauder and J. A. Wheeler, Rev. Mod. Phys. 29, 516 (1957); T. M. Davis and J. R. Ray, J. Math. Phys. 16, 75 (1975), Phys. Rev. D 9, 331 (1974), J. Math. Phys. 16, 80 (1975); K. D. Kriori and H. Kakati, GRG 20, 1237 (1995); J. C. Huang, N. O. Santos and Kleber, Class. Quantum Grav. 12, 1245 (1995); I. D. Soares and J. Tiomno, Phys. Rev. D 54, 2808 (1996); C. G. De Oliveira and J. Tiomno, Il Nouvo Cimento 24, 672 (1962); B. D. B. Figueredo, I. D. Soares and Tiomno, Class. Quantum Grav. 9, 1593 (1992); Hammond R., Class. Quantum Grav. 12, 279 (1995); P. Baekler, M. Setz, V. Winkelmann, Class. Quantum Grav. 5, 479 (1988); V. M. Villalba, Mod. Phys. Lett. A 8, 2351 (1993)

[15] S. A. Pol'shin, hep-th/0001040; hep-th/0001069 
[16] R. Gilmore, Lie Groups, Lie Algebras and Some of Their Applications (Wiley-Interscience, New York, 1974)

[17] M. Hamermesh, Group theory and its applications to physical problems (Addison-Wesley, Reading MA, 1962)

[18] A. O. Barut and R. Raçzka, Theory of Group Representations and Applications (PWN, Warszawa, 1977)

[19] G. Mackey, Induced Representations of Groups and Quantum Mechanics (Benjamin, New York, 1968)

[20] V. M. Villalba, Eur. J. Phys. 15, 191 (1994) 